\title{
ON UNIFORM LATTICES IN REAL SEMISIMPLE GROUPS
}

\author{
CHANDRASHEEL BHAGWAT AND SUPRIYA PISOLKAR
}

(Communicated by Lev Borisov)

\begin{abstract}
In this article we prove that the co-compactness of the arithmetic lattices in a connected semisimple real Lie group is preserved if the lattices under consideration are representation equivalent. This is in the spirit of the question posed by Gopal Prasad and A. S. Rapinchuk in 2014 where instead of representation equivalence, the lattices under consideration are weakly commensurable Zariski dense subgroups.
\end{abstract}

\section{INTRODUCTION}

In 9, G. Prasad and A. S. Rapinchuk defined the notion of weakly commensurable Zariski dense subgroups in absolutely almost simple algebraic groups. Among many other striking implications of this seemingly weak notion they have proved that weakly commensurable subgroups in the group of rational points of absolutely almost simple algebraic groups determine the type of the group except in the case when one is of type B and the other of type C. They show that length commensurable arithmetic lattices are weakly commensurable. For this, when the locally symmetric spaces are of rank greater than 1, they assume the validity of Schanuel's conjecture. Further using methods from arithmetic theory of algebraic groups, they obtain commensurability type results for isospectral compact locally symmetric spaces.

In [1, we assumed the stronger hypothesis that the lattices defining the locally symmetric spaces are representation equivalent rather than isospectral on functions. This allowed us to obtain similar conclusions as in [9] for representation equivalent lattices, without invoking Schanuel's conjecture.

In the sequel to their work on weakly commensurable subgroups [10, Prasad and Rapinchuk posed the following question. For $i=1,2$, let $G_{i}$ be a connected absolutely almost simple group defined over $F=\mathbb{R}$ or $\mathbb{C}$ and let $\Gamma_{i}$ be a lattice in $G_{i}(F)$. Assume that $\Gamma_{1}$ is weakly commensurable to $\Gamma_{2}$. Does compactness of $\Gamma_{1} \backslash G_{1}(F)$ imply the compactness of $\Gamma_{2} \backslash G_{2}(F)$ ? When the corresponding locally symmetric spaces are length commensurable and one of the spaces is arithmetically defined, Theorem 6 and Theorem 7 of $[9]$ provide an affirmative answer to the above question. We recall that the co-compactness of a lattice in a semisimple real Lie group is equivalent to the absence of nontrivial unipotents in it (cf. [11, Corollary 11.13]). Thus the above question can be rephrased as whether for two weakly

Received by the editors August 20, 2015.

2010 Mathematics Subject Classification. Primary 22E45; Secondary 22E40, 11M36, 11F72.

The first author was partially supported by DST-INSPIRE Faculty scheme, award number [IFA- 11MA-05]. 
commensurable lattices, the existence of nontrivial unipotent elements in one of them implies their existence in the other.

In this article we address a similar question under the stronger hypothesis of representation equivalence. We prove that:

Theorem 1.1. Let $G$ be a connected semisimple real Lie group. Let $\Gamma_{1}, \Gamma_{2}$ be representation equivalent arithmetic lattices in $G$. Then $\Gamma_{1} \backslash G$ is compact if and only if $\Gamma_{2} \backslash G$ is compact.

Remark 1.2. By the arithmeticity theorem of Margulis, if $G$ is a real semisimple algebraic group without compact factors and such that $\mathbb{R}$-rank of $G$ is $\geq 2$, then every irreducible lattice is arithmetic. If $\mathbb{R}$-rank is 1 , a result of Corlette in the archimedean case and of Gromov-Schoen in the nonarchimedean case shows that lattices in $S p(n, 1), n \geq 2$, and $F_{4}^{-20}$ are arithmetic.

Remark 1.3. For $p$-adic groups, Theorem 1.1 is a tautology since every lattice is co-compact.

\section{Preliminaries}

2.1. Lattices and representation equivalence. Let $G$ be a connected semisimple real Lie group. Suppose $\Gamma$ is a discrete subgroup of $G$ such that the quotient $\Gamma \backslash G$ has a finite $G$-invariant Borel measure $\mu$. Consider the space $L^{2}(\Gamma \backslash G)$ of all complex valued measurable $\Gamma$-invariant functions on $G$ such that

$$
\int_{\Gamma \backslash G}|f(x)|^{2} d \mu(x)<\infty .
$$

The right regular representation $R_{\Gamma}$ of $G$ is on $L^{2}(\Gamma \backslash G)$, defined by

$$
R_{\Gamma}(g) f(x)=f(x g) \quad \forall g, x \in G \text { and } f \in L^{2}(\Gamma \backslash G) .
$$

It is well known that this defines a unitary representation of $G$ on the Hilbert space $L^{2}(\Gamma \backslash G)$.

Let $\widehat{G}$ be the set of all equivalence classes of irreducible unitary representations of $G$. We will denote an element of $\widehat{G}$ by $\omega$. We now recall the following result (cf. [12, 14.10.5]) which describes the direct integral decomposition of $R_{\Gamma}$ with respect to the irreducible unitary representations of the group $G$.

Theorem 2.1. Let $(\pi, \mathcal{H})$ be a unitary representation of $G$ on a Hilbert space $\mathcal{H}$. There exists a Borel measure $\sigma$ on $\widehat{G}$ and a family of unitary representations $\left(\pi_{\omega}, H_{\omega}\right)$ such that:

(1) The representation $(\pi, \mathcal{H})$ is unitarily equivalent to a direct integral as follows:

$$
(\pi, \mathcal{H}) \cong \int_{\widehat{G}}\left(\pi_{\omega}, H_{\omega}\right) d \sigma(\omega) .
$$

(2) Each $\left(\pi_{\omega}, H_{\omega}\right)$ is unitarily equivalent to the Hilbertian tensor product $\left(\pi^{\prime}{ }_{\omega} \otimes I, H^{\prime}{ }_{\omega} \otimes V_{\omega}\right)$ of an irreducible unitary representation $\left(\pi^{\prime}{ }_{\omega}, H^{\prime}{ }_{\omega}\right) \in \omega$ and the trivial $G$ representation $I$ on some Hilbert space $V_{\omega}$.

(3) The map $\omega \mapsto \operatorname{dim}\left(V_{\omega}\right)$ is measurable w.r.t. the measure $\sigma$.

The following result from [3] gives the appropriate uniqueness for the measure $\sigma$ in Theorem 2.1. 
Proposition 2.2. If there are two Borel measures $\sigma$ and $\mu$ on $\widehat{G}$ such that all the three conditions in Theorem 2.1 hold, then $\sigma$ and $\mu$ are mutually absolutely continuous, i.e., for any Borel set E,

$$
\sigma(E)=0 \Leftrightarrow \mu(E)=0
$$

2.2. Eisenstein series. In this subsection we recall some of the relevant facts from the theory of Eisenstein series from Langlands' work [5], 6] and [8]. In particular we discuss the decomposition of the Hilbert space $L^{2}(\Gamma \backslash G)$ into certain $G$-invariant spaces parametrized by various parabolic subgroups.

Let $G$ be the group of real points of a connected semisimple group $\mathbf{G}$ defined over $\mathbb{Q}$ and let $\Gamma$ be a lattice in $G$ which we assume to be neat. Let us fix a minimal parabolic subgroup $\mathbf{P}$ of $\mathbf{G}$ defined over $\mathbb{Q}$ and a maximal $\mathbb{Q}$-split torus $\mathbf{A}$ of $\mathbf{P}$. A standard cuspidal parabolic subgroup $P$ is the normalizer of a parabolic subgroup $\mathbf{P}$.

Let $\mathfrak{a}_{\mathbb{C}}$ be the complexification of the Lie algebra of real points of $\mathbf{A}$ and let $\mathfrak{a}$ be the set of real points of $\mathfrak{a}_{\mathbb{C}}$ corresponding to the split component of $P$. Consider a decomposition $P=A M N$ of $P$, where $A=\mathbf{A}_{\mathbb{R}}^{o}$ is the analytic subgroup of $G$ with Lie algebra $\mathfrak{a}, N$ is the unipotent radical of $P$ and $M$ is a reductive group identified with $N \backslash M N$. Since $\Gamma$ is neat, $\Gamma \cap P \subseteq M N$ and $\Theta:=\Gamma \cap N \backslash \Gamma \cap M N$ can be thought of as a subgroup of $N \backslash M N \cong M$. Let $S=M N$. Let $(P, S)$ and $\left(P^{\prime}, S^{\prime}\right)$ be two split parabolic subgroups of $G$. Then we say that $(P, S)$ is a successor of $\left(P^{\prime}, S^{\prime}\right)$, i.e., $(P, S) \geq\left(P^{\prime}, S^{\prime}\right)$ if $P \supset P^{\prime}$ and $S \supset S^{\prime}$. Further $(P, S)$ is called a dominant successor of $\left(P^{\prime}, S^{\prime}\right)$ if there exists a chain

$$
(P, S)=\left(P_{1}, S_{1}\right) \geq\left(P_{2}, S_{2}\right) \geq \cdots \geq\left(P_{n}, S_{n}\right)=\left(P^{\prime}, S^{\prime}\right)
$$

such that

$$
\begin{gathered}
P_{1} \supseteq P_{2} \supseteq \cdots \supseteq P_{n}, \\
A_{1} \subseteq A_{2} \subseteq A_{2} \subseteq \cdots \subseteq A_{n}
\end{gathered}
$$

and $\operatorname{dim}\left(A_{i+1}\right)-\operatorname{dim}\left(A_{i}\right)=1,1 \leq i \leq n$.

Definition 2.3. A subgroup $(P, S)$ is said to be $\Gamma$-cuspidal if every dominant successor $\left(P^{\prime}, S^{\prime}\right)$ of $(P, S)$ has the following properties:

(1) $\Gamma \cap P^{\prime}$ is contained in $S^{\prime}$.

(2) $N^{\prime} / N^{\prime} \cap \Gamma$ is compact.

(3) $S^{\prime} / S^{\prime} \cap \Gamma$ is of finite volume.

If, moreover, $S / S \cap \Gamma$ is compact, then $(P, S)$ is said to be $\Gamma$ per-cuspidal.

Let $E(G, \Gamma)$ denote the set of all $\Gamma$-per-cuspidal subgroups of $G$. We recall here the important result about $E(G, \Gamma)$ (cf. [8, Proposition 2.6])

Proposition 2.4. Modulo $\Gamma$-conjugacy, there are only finitely many elements of $E(G, \Gamma)$.

The number of cusps of $\Gamma$ is then, by definition, $|(\Gamma \backslash E(G, \Gamma))|$.

Definition 2.5. Two cuspidal subgroups $P$ and $P^{\prime}$ are said to be associate if there is an element of the Weyl group which takes $\mathfrak{a}_{C}$ to $\mathfrak{a}_{C}^{\prime}$.

Consider a decomposition $P=A M N$ of $P$ as before and denote by $Z$ the center of the universal enveloping algebra of $M$. Let $V(\xi):=\left\{\phi \in L_{0}^{2}(\Theta \backslash M): X \phi=\right.$ $\xi(X) \phi \quad \forall X \in Z\}$ for $\xi \in \operatorname{Hom}(Z, \mathbb{C})$. 
Let $E$ be the set of all orbits of the action of $Z$ on $\operatorname{Hom}(Z, \mathbb{C})$ and let $V_{E}:=$ $\bigoplus_{\xi \in E} V(\xi)$. This is a closed $M$-invariant subspace of $L_{0}^{2}(\Theta \backslash M)$ such that

$$
L_{0}^{2}(\Theta \backslash M)=\bigoplus_{E} V_{E}
$$

Such a $V_{E}$ is called a simple admissible subspace of $L_{0}^{2}(\Theta \backslash M)$.

Fix such a simple admissible subspace $V$. Let $K$ be a maximal compact subgroup of $G$ and let $W$ be the space spanned by the matrix coefficients of some irreducible representation of $K$.

Let $\mathcal{D}(V, W)$ be the space of all continuous functions $\phi$ on $N(\Gamma \cap P) \backslash G$ such that $m \mapsto \phi(m g)$ belongs to $V$ and $k \mapsto \phi\left(g k^{-1}\right)$ belongs to $W$ for all $g \in G$ and such that the support of $\phi$ on $N M \backslash G$ is compact.

Let $\{P\}$ be an associate class of per-cuspidal parabolic subgroups of $G$. Define $L(\{P\},\{V\}, W)$ to be the closed subspace spanned by functions $\hat{\phi}$ with $\phi \in$ $\mathcal{D}(V(P), W)$ for some $P \in\{P\}$.

From Lemma 2 in [5, we know that:

Lemma 2.6. The space $L^{2}(\Gamma \backslash G)$ is the orthogonal direct sum of the spaces as follows:

$$
L^{2}(\Gamma \backslash G)=\bigoplus_{\{P\}} L(\{P\},\{V\}, W) .
$$

Further, each $L(\{P\},\{V\}, W)$ can be decomposed as:

$$
L(\{P\},\{V\}, W)=\bigoplus_{i=0}^{g} L_{i}(\{P\},\{V\}, W)
$$

where $g$ is the common rank of all parabolic subgroups in the class $\{P\}$.

Remark 2.7. (1) The important hypothesis about the lattice $\Gamma$ for the above result as in [6] was that $\Gamma$ possesses a fundamental domain. It follows from the results of Raghunathan and Garland [4 in the rank one case and of Margulis [7 in the higher rank case that there exist fundamental domains for the arithmetic lattices in $G$. Thus the hypotheses in the decomposition theorem of Langlands (as in [5], 6]) are satisfied.

(2) In his result in [6], Langlands considers a complete set $\mathcal{P}(G, \Gamma)$ of per-cuspidal subgroups of $G$. It can be verified (cf. [8, page 78]) that the set $E(G, \Gamma)$ is exactly the set $\mathcal{P}(G, \Gamma)$.

\section{MAin Results}

In this section we prove the main result of this article.

Theorem 3.1. Let $G$ be a connected semisimple real Lie group and let $\Gamma_{1}, \Gamma_{2}$ be two arithmetic lattices in $G$. If the lattices $\Gamma_{1}, \Gamma_{2}$ are representation equivalent and $\Gamma_{1} \backslash G$ is compact, then $\Gamma_{2} \backslash G$ is also compact.

The main ingredient of the proof of this theorem is the following characterization of co-compact lattices in real semisimple Lie groups. 
Theorem 3.2. Let $\Gamma$ be an irreducible lattice in $G$. Then the quotient $\Gamma \backslash G$ is compact if and only if the direct integral decomposition given by Theorem 2.1 is a Hilbert direct sum, i.e.,

$$
R_{\Gamma} \cong \widehat{\bigoplus_{j \in \mathbb{N}}}\left(\pi_{j}, V_{j}\right)
$$

for a countable family of irreducible unitary representations $\left(\pi_{j}, V_{j}\right)_{j \in \mathbb{N}}$ such that each $\pi_{j}$ occurs with a finite multiplicity.

Proof. If $\Gamma \backslash G$ is compact, then it is well known that $R_{\Gamma}$ is a Hilbert direct sum as required. Conversely, if $\Gamma$ is not uniform, then there is a unipotent element $u \neq 1$ in $\Gamma$ (cf. [11, Corollary 11.13]). To such a unipotent element $u$ in $\Gamma$ one can associate a proper parabolic subgroup of $G$. Indeed, when $\mathbf{G}$ is of $\mathbb{Q}$-rank 1 , then by [11, cf. 12.17], $u$ is contained in a unique $\mathbb{Q}$-parabolic subgroup of $\mathbf{G}$. In the case when $\mathbb{Q}$-rank of $\mathbf{G}$ is at least 2 , one associates to $u$ a $\mathbb{Q}$-parabolic subgroup $P_{u}$ of $\mathbf{G}$ containing $u$ by following the procedure of Borel-Tits in [2]. Let $U_{1}$ be the one-parameter subgroup containing $u$, then take its normaliser $N_{1}$. Let $U_{2}$ be the unipotent radical of $N_{1}$ (it contains $U_{1}$ ). Let $N_{2}$ be the normaliser of $U_{2}$. After some stage this chain of $\mathbb{Q}$-subgroups $N_{i}$ and $U_{i}$ stabilizes. Thus, we get the unipotent group $U=U_{n}$ which is the unipotent radical of the normaliser $P=N_{n}$, and $U_{n}=U_{n+1}$. Then a result of Borel-Tits (cf. [2]) says that $P_{u}:=P$ is a proper parabolic subgroup containing $U \supset U_{1}$ and $u \in U_{1}$.

Let $E(G, \Gamma)$ be the set of all per-cuspidal parabolic subgroups of $G$ as in section 2.2. Since $\Gamma$ is arithmetic, $P_{u} \in E(G, \Gamma)$ (cf. [8, pp. 23, 63]. Thus $P_{u}$ appears in the decomposition in Lemma 2.6. It follows from [5, p. 252] that the space $L_{i}(\{P\},\{V\}, W)$ has a continuous spectrum of dimension $i$. Thus we conclude that there is a nontrivial continuous spectrum in the above decomposition since $P$ is proper. Hence the result follows.

We now give the proof of the main theorem.

Proof of Theorem 3.1. The lattice $\Gamma_{1}$ is co-compact, so by Theorem 2.1 there is a countable subset $E$ of $\widehat{G}$ such that the support of $\mu_{1}$ equals $E$. (Recall that the support of a measure is the set of all points $\omega$ in $\widehat{G}$ for which every open neighborhood $U$ of $\omega$ has positive measure.)

Let $\mu_{1}$ and $\mu_{2}$ be the measures on $\widehat{G}$ corresponding to the representations $R_{\Gamma_{1}}$ and $R_{\Gamma_{2}}$, respectively. Hence the measures $\mu_{1}$ and $\mu_{2}$ are mutually absolutely continuous by Proposition 2.2. It follows that their supports are equal. Thus the support of the measure $\mu_{2}$ also equals $E$. In other words, $R_{\Gamma_{2}}$ has a decomposition as a Hilbert direct sum of irreducible representations of $G$. From Theorem 3.2 the desired result follows.

\section{ACKNOWLEDGEMENTS}

The authors thank M. S. Raghunathan, Nolan Wallach, Laurent Clozel, David Vogan, Paul Garrett, A. Raghuram and Sandeep Varma for their interest and helpful discussions. The authors offer their sincere thanks to Gopal Prasad for pointing out the inaccuracies in a previous version. 


\section{REFERENCES}

[1] Chandrasheel Bhagwat, Supriya Pisolkar, and C. S. Rajan, Commensurability and representation equivalent arithmetic lattices, Int. Math. Res. Not. IMRN 8 (2014), 2017-2036. MR3194011

[2] A. Borel and J. Tits, Éléments unipotents et sous-groupes paraboliques de groupes réductifs. I (French), Invent. Math. 12 (1971), 95-104. MR0294349 (45 \#3419)

[3] Jacques Dixmier, Les $C^{*}$-algèbres et leurs représentations (French), Deuxième édition. Cahiers Scientifiques, Fasc. XXIX, Gauthier-Villars Éditeur, Paris, 1969. MR0246136 (39 \#7442)

[4] H. Garland and M. S. Raghunathan, Fundamental domains for lattices in (R-)rank 1 semisimple Lie groups, Ann. of Math. (2) 92 (1970), 279-326. MR0267041 (42 \#1943)

[5] R. P. Langlands, Eisenstein series, Algebraic Groups and Discontinuous Subgroups (Proc. Sympos. Pure Math., Boulder, Colo., 1965), Amer. Math. Soc., Providence, R.I., 1966, pp. 235-252. MR0249539 (40 \#2784)

[6] R. P. Langlands, On the functional equations satisfied by Eisenstein series, Lecture Notes in Mathematics, Vol. 544, Springer-Verlag, Berlin-New York, 1976. MR0579181 (58 \#28319)

[7] G. A. Margulis, On the arithmeticity of discrete groups (Russian), Dokl. Akad. Nauk SSSR 187 (1969), 518-520; English transl., Soviet Math. Dokl. 10 (1969), 900-902. MR0293007 (45 \#2088)

[8] M. Scott Osborne and Garth Warner, The theory of Eisenstein systems, Pure and Applied Mathematics, vol. 99, Academic Press, Inc. [Harcourt Brace Jovanovich, Publishers], New York-London, 1981. MR643242 (83j:10034)

[9] Gopal Prasad and Andrei S. Rapinchuk, Weakly commensurable arithmetic groups and isospectral locally symmetric spaces, Publ. Math. Inst. Hautes Études Sci. 109 (2009), 113184, DOI 10.1007/s10240-009-0019-6. MR2511587 (2010e:20074)

[10] Gopal Prasad and Andrei S. Rapinchuk, Generic elements in Zariski-dense subgroups and isospectral locally symmetric spaces, Thin groups and superstrong approximation, Math. Sci. Res. Inst. Publ., vol. 61, Cambridge Univ. Press, Cambridge, 2014, pp. 211-252. MR3220892

[11] M. S. Raghunathan, Discrete subgroups of Lie groups, Ergebnisse der Mathematik und ihrer Grenzgebiete, Band 68, Springer-Verlag, New York-Heidelberg, 1972. MR0507234 $(58$ \#22394a)

[12] Nolan R. Wallach, Real reductive groups. II, Pure and Applied Mathematics, vol. 132, Academic Press, Inc., Boston, MA, 1992. MR1170566 (93m:22018)

Indian Institute of Science Education And Research, Pune 411008, India

E-mail address: cbhagwat@iiserpune.ac.in

Indian Institute of Science Education and Research, Pune 411008, India

E-mail address: supriya@iiserpune.ac.in 